EPJ Web of Conferences 47, 06005 (2013)

DOI: $10.1051 /$ epjconf/20134706005

(C) Owned by the authors, published by EDP Sciences, 2013

\title{
The WFCAM transit survey and cool white dwarfs
}

\author{
S. Catalan ${ }^{1, a}$, R. Napiwotzki ${ }^{1}$, S. Hodgkin ${ }^{2}$, D. Cristobal Hornillos ${ }^{3}$ \\ and D. Pinfield ${ }^{1}$ \\ ${ }^{1}$ Centre for Astrophysics Research, University of Hertfordshire, Hatfield AL 10 9AB, UK \\ 2 Institute of Astronomy, Madingley Road, Cambridge CB3 OHA, UK \\ ${ }^{3}$ Centro de Estudios de Fisica del Cosmos de Aragon (ceFca), 44001 Teruel, Spain
}

\begin{abstract}
We present results from our search for cool white dwarfs in the WTS (WFCAM Transit Survey). Repeat observations starting in 2007 allowed to produce deep stacked images in $\mathbf{J}$ and measure proper motions. We combine this with deep optical imaging to select cool white dwarf candidates $\left(T_{\text {eff }}<5000 \mathrm{~K}\right)$. About 27 cool white dwarf candidates with proper motions above $0.10 \mathrm{arcsec} / \mathrm{yr}$ were identified in one of the fields representing 1/8th of the survey area. Follow-up spectroscopy with the $10.2 \mathrm{~m}$ GTC telescope at La Palma confirmed the white dwarf status for all observed candidates. On-going work is being carried out to increase the sample of cool white dwarfs that will allow a more comprehensive study of the thick disk/halo white dwarf population.
\end{abstract}

\section{INTRODUCTION}

The study of the oldest white dwarfs can provide relevant information about the early star formation of the Galaxy and its initial-mass function. A number of so-called ultra-cool white dwarfs ( $T_{\text {eff }}<4000 \mathrm{~K}$ ) that show flux depression in the IR due to hydrogen molecule absorption have been detected so far [3]. However, it is believed that most or all of these ultra-cool white dwarfs are low-mass products of binary evolution and thus not representative for the oldest white dwarfs. Their low mass causes relatively high luminosity making them the first cool white dwarfs detected in relatively shallow surveys. Deeper observations are needed for the oldest, high mass white dwarfs with the longest cooling times.

[10] reported a very large population of white dwarfs belonging to the galactic halo in line with a top heavy initial mass function. This result was disputed by [12] and [11], but all these investigations are based on very local samples and large uncertainties remain, because of small number statistics. A particular problem is the lack of confirmed very cool population II white dwarfs (the first produced in the Galaxy), which are most interesting, because they evolved from the most massive progenitor stars. Even the population I white dwarf luminosity function constructed by [2], comprising of 6000 WDs selected from the Sloan Digital Sky Survey (SDSS) contains only 35 cool WDs with absolute magnitudes $>15$ mag. In a recent work, [6] identified few cool white dwarfs using SDSS data, although only a couple have proper motions compatible with the halo population [7].

\section{IMAGING DATA AND PROPER MOTION CALCULATION}

We participate in a project called WTS (WFCAM Transit Survey), - co-PI'ed by D. Pinfield and S. Hodgkin. The aim of this survey is the detection of planetary companions to low-mass stars from

\footnotetext{
${ }^{a}$ e-mail: s.catalan@herts . ac.uk
}

This is an Open Access article distributed under the terms of the Creative Commons Attribution License 2.0, which permits unrestricted use, distribution, and reproduction in any medium, provided the original work is properly cited. 


\section{EPJ Web of Conferences}
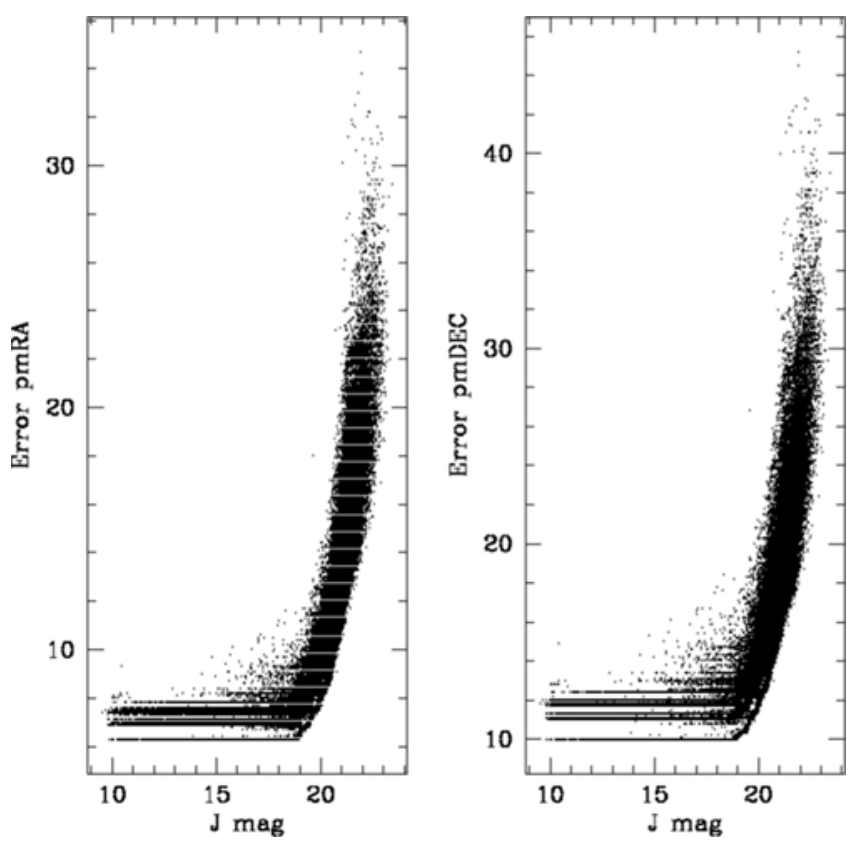

Figure 1. Errors in proper motions versus J band magnitude.

the transit light curves. This survey is carried out at the UKIRT telescope using the $Z Y J H K$ filters. WTS will take over the time span of five years many repeat observations in NIR bands with a total coverage of 6 sq. deg. (4 fields). Observations of the four fields are expected to be completed by 2012. The $J$ band data will be the deepest with a total of 25 hours spent on each field (see Sipocz et al. 2013, this volume). The $Z Y H K$ data have a depth of 19-21, so these are useful for bright objects. In order to be able to obtain deep images but still have good accuracy in proper motion we decided to co-add $\mathbf{J}$ band images corresponding to same month (30-40 images). We only included those images that fulfilled some requirements: seeing $<1.2$ arcsec, airmass $<1.5$ and zeropoint $>22.8$.

To compute the proper motions we use the J-band stacked images from different epochs, since faint objects would not appear in the single J-band images. We pick the first image (first epoch) as a reference, and then follow each detected star through all the other images. The stars are cross-matched on a plate-by-plate basis (CCD) to compute the offsets. The typical error obtained in right ascension and declination is approx. 30 mas for bright stars, going up to 50-60 mas close to the limiting magnitude. These errors have been computed from the scatter of the individual measurements around the solutions with proper motion. In Fig. 1 we show the errors in proper motions versus $\mathbf{J}$ band magnitude.

In order to cover the SED of possible white dwarf candidates, we used the $3.5 \mathrm{~m}$ telescope at Calar Alto Observatory with the LAICA instrument and three SDSS filters (g, r, i bands) obtaining a depth of $r=24 \mathrm{mag}(5 \sigma)$. We have managed to completely cover 3 of the 4 WTS fields. The results presented here correspond to half of the $19 \mathrm{~h}$ field. The LAICA image scale is $0.225 \mathrm{arcsec} / \mathrm{pixel}$ and its field of view is $44.36 \times 44.36 \mathrm{arcmin}$. The detector consists in $2 \times 2$ mosaic of $4 k \times 4 k$ CCDs. The size of each CCD is 15.36 arcmin and the gap between the CCDs is of 13.64 arcmin. Thus, in order to completely cover one of the WTS field $(0.75 \times 1.5 \mathrm{deg}) 8$ pointings are necessary. For the initial processing of the images we have used an adapted version of the pipeline of the Alhambra Survey project, which used the same telescope and instrument as in this work [8]. The photometric calibration has been done using the corresponding IRAF tasks. 


\section{Hot Planets and Cool Stars}
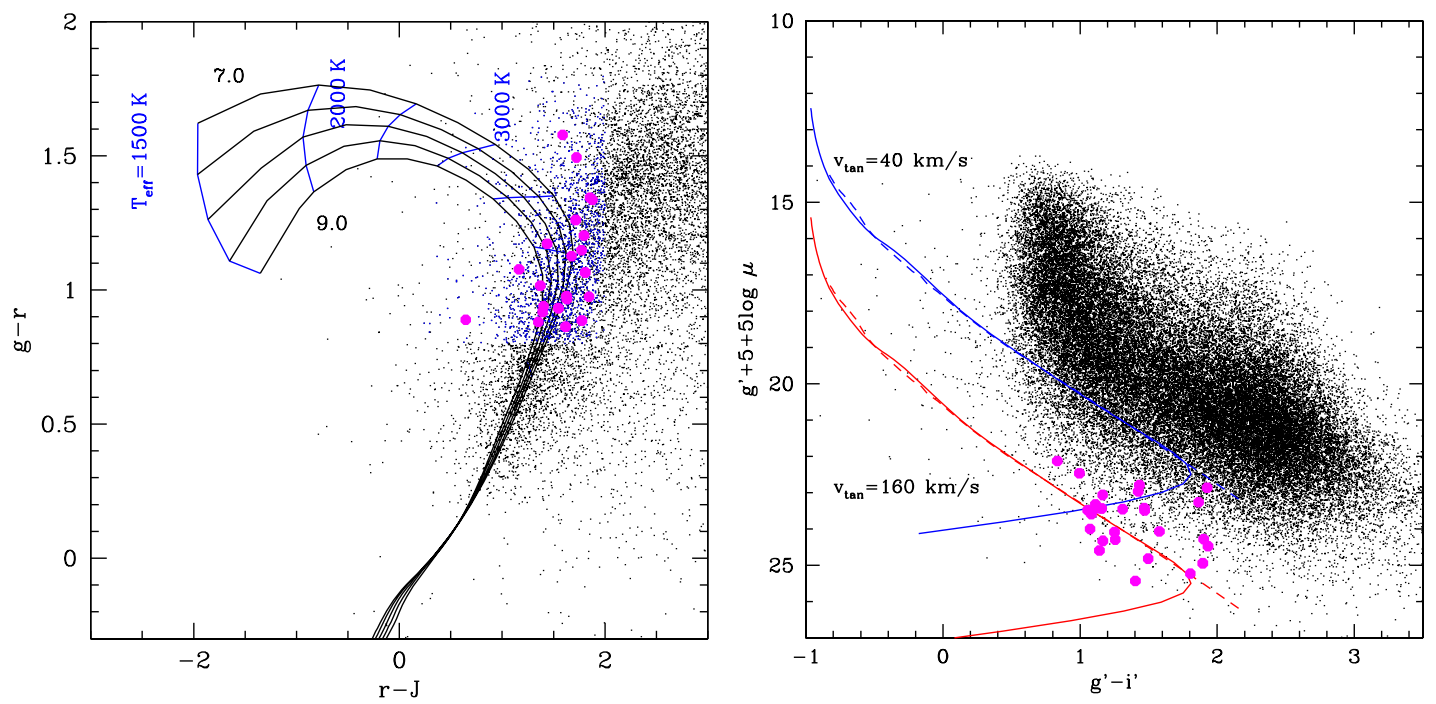

Figure 2. Left: colour-colour diagram for the objects detected in our survey (black points). Solid circles (magenta) correspond to high-proper motion white dwarf candidates with $\mu>0.1 \mathrm{arcsec} / \mathrm{yr}$. The grid corresponds to the improved synthetic photometry by [4]. Right: reduced proper motion diagram. Solid circles mean the same as in left. The solid lines correspond to the theoretical tracks for H composition for $v_{\text {tan }}=40 \mathrm{~km} / \mathrm{s}$ (top) and $160 \mathrm{~km} / \mathrm{s}$ (bottom). The dashed lines correspond to He composition. Note that some of our white dwarf candidates could have mixed compositions too.

\section{COOL WHITE DWARF IDENTIFICATION}

From the combination of optical + IR photometry and the ppms $(\mu)$ we have been able to identify thick disc/halo white dwarf candidates. In Fig. 2 (left) we show a colour-colour diagram for the stellar objects detected in our survey (black points) in the half of the 19h WTS field. Solid circles (magenta) correspond to the high-proper motion cool white dwarf candidates $(\mu>0.1 \mathrm{arcsec} / \mathrm{yr})$ identified. These candidates were obtained from applying the following colour cuts to select cool white dwarf candidates: $0.5<g-r<1.8,-2.6<r-i<0.6, g-i<2.2$ and $J>14$. The reduced proper motion $\left(H_{g}=g+5+5 \log \mu\right)$ is a very useful parameter to identify thick disc/halo white dwarf candidates. In Fig. 2 (right) we show the reduced proper motion diagram that we obtained using our recent data. We have identified 27 candidates that meet the white dwarf colour cuts and the reduced proper motion criteria $H_{g}>15.136+2.727(g-i)$ and have high proper motion $(\mu>0.1 \operatorname{arcsec} / \mathrm{yr})$, indicating that they belong to the thick disc-halo populations.

We have obtained $T_{\text {eff }}$ from a fit of the available photometry (griJ) to synthetic magnitudes confirming that the objects are colder than $5000 \mathrm{~K}$. We considered both pure-H and pure-He compositions. The pure-H models used are those of [14], but now including the red wing opacity from Ly $\alpha$. The pure-He model atmospheres considered are the same models as those used in [5]. The surface gravity is fixed to $\log g=8$ (the most typical value for white dwarfs). We performed follow-up spectroscopy with the $10.2 \mathrm{~m}$ GTC telescope at La Palma of 3 objects, confirming the white dwarf status. More observing time is needed to confirm the other candidates.

\section{CONCLUSIONS}

In this work we have presented a follow-up of our project to search for the coolest white dwarfs in the Galaxy. We have obtained proper motions for the objects in the $19 \mathrm{~h}$ field using stacked $\mathrm{J}$ band 
images from the WTS survey. From the combination of NIR and optical observations, together with the proper motion information we have identified 27 white dwarf candidates with $\mu>0.1 \mathrm{arcsec} / \mathrm{yr}$. We have spectroscopically confirmed three of these objects.

In the near future our main objective will be to complete the optical imaging of the four WTS fields. A comparison of the obtained luminosity function and the white dwarf population simulations of [9] will allow us to obtain information about the IMF. We will continue with the spectroscopic follow-up of the coolest white dwarf candidates to confirm their classification and study the atmospheric properties. The identification and characterization of cool white dwarfs in this project will have an impact on other fields of astrophysics: Hydrogen opacities are rather uncertain at low temperatures. Theoretical work is going on for further improvement (e.g. [13]) but observational benchmarks are important. Note hydrogen have an impact on the IR flux of brown dwarfs as well, which are observed down to much lower temperatures than white dwarfs [1]. We will obtain accurate observational SEDs of cool white dwarfs that will give an unprecedented input to test the theoretical models.

\section{References}

[1] Allard, F., Hauschildt, P.H., Alexander, D.R. et al., ApJ 556, 357 (2001)

[2] Harris, H.C., Munn, J.A., \& Kilic, M., AJ 131, 571 (2005)

[3] Harris, H.C., Gates, E. et al., ApJ 679, 697 (2008)

[4] Holberg, J. \& Bergeron, P., AJ 132, 1221 (2006)

[5] Kilic, M., Leggett, S. K., Tremblay, P.-E., et al., ApJS 190, 77 (2010a)

[6] Kilic, M., Munn, J. A., Williams, K. A., et al., ApJ, 715, L21 (2010b)

[7] Leggett, S.K., Lodieu, N., Tremblay, P.E., Bergeron, P. \& Nitta, A., ApJ, 735, 62 (2011)

[8] Moles M., Benitez, N., Aguerri, J.A.L., et al., AJ 136, 1325 (2008)

[9] Napiwotzki, R., JPCS 172, 2004 (2009)

[10] Oppenheimer, B.R., Hambly, N.C., Digby, A.P., et al., Science 292, 698 (2001)

[11] Pauli, E.M., Napiwotzki, R., Heber, U., et al., A\&A 447, 173 (2006)

[12] Reid, I., Sahu, K., \& Hawley, S.L., ApJ 559, 942 (2001)

[13] Tremblay, P.E., \& Bergeron, P., ApJ 696, 1755 (2009)

[14] Tremblay, P.E., Bergeron, P. \& Gianninas, A., ApJ 730, 128 (2011) 